\title{
Rapid diagnosis of Gram negative urinary infections: identification and antimicrobial susceptibility testing
} in 24 hours

\author{
CATHERINE M DUPEYRON, GEORGIE A GUILLEMIN, GJ LELUAN
}

From the Henri Mondor Hospital Group, Albert Chenevier Bacteriology Laboratory, Albert Chenevier Hospital, Creteil, France

SUMMARY A rapid method for diagnosing urinary tract infections, using identification and antimicrobial susceptibility testing that can be carried out in 24 hours, was devised. The method relies on direct inoculation of diluted urine (1/500) in the API 20 E and API ATB systems. Urine was simultaneously cultured on Columbia blood agar and on Drigalski agar to control the purity and for purposes of comparison.

The results of this method and those obtained with a conventional method were compared by analysing 1352 urines. The results showed that all of the organisms were correctly identified using the conventional method, and susceptibility testing (rapid method) gave results that agreed with those of the classical method in $94 \%$ of cases, with major discrepancies in only $0.08 \%$ of cases. The rapid method applies only to monomicrobial infections.

Systems enabling identification and antimicrobial susceptibility testing of enterobacteria within a few hours are now common, and some authors have described their use in the direct analysis of urine. In general, satisfactory results were obtained, but expensive equipment was required. ${ }^{1-6}$ It is obviously desirable to develop rapid and sensitive direct testing methods, which are reliable but inexpensive and which would yield results within 24 hours. For that purpose we used the API 20 system for enterobacteria (API $20 \mathrm{E})^{7-9}$ and the API system for antimicrobial susceptibility testing (ATB Gram negative). ${ }^{10}$

The aim of this study was to compare the results obtained with the direct method with those obtained using a classical method.

\section{Material and methods}

Urine specimens (1352) were obtained from inpatients with various diseases, who required routine urine cytobacteriological examination. The urines obtained aseptically were kept at $4^{\circ} \mathrm{C}$ in closed sterile containers. A maximum delay of two hours was tolerated between the time of collection and the analysis of the specimens. The cytological examination was per-

Accepted for publication 14 October 1985 formed in a haemocytometer and included leucocyte, red cell, and epithelial cell counts. A Gram stain was done on non-centrifuged urine. All the urines were inoculated according to the usual procedure of our laboratory (conventional method). The urines containing Gram negative bacilli (on direct examination) but no cocci were treated simultaneously with the rapid method.

\section{CONVENTIONAL METHOD}

Urine $(50 \mu \mathrm{l})$, diluted $1 / 100$ in sterile distilled water was plated on Columbia agar enriched with horse blood and on Drigalski agar distributed in Petri dishes. The inoculum was spread with a glass rake over one half of each dish, and a streak isolation was performed on the second half. After 18 to 24 hours of incubation a colony count was performed on the first half of the Columbia blood agar dish: the amount of urine plated permitted a rapid determination-104 bacteria $/ \mathrm{ml}$ yielded five colonies, $10^{5}$ bacteria $/ \mathrm{ml}$ yielded 50 colonies, and $10^{6}$ bacteria $/ \mathrm{ml}$ yielded innumerable colonies. Isolates obtained from the second half of the dishes were used for identification and antimicrobial susceptibility testing with the API $20 \mathrm{E}$ and ATB Gram negative systems. Identification and antimicrobial susceptibility testing were always performed with the same suspension, which had been obtained from a single colony and diluted according to the manufacturer's instructions. 


\section{RAPID METHOD}

The urines containing Gram negative bacilli (on direct examination) but no cocci were selected and analysed using the rapid method. Two hundred of the 1352 specimens were studied with this protocol to evaluate the method. The API 20 E system was inoculated with a $1 / 500$ dilution of urine, and 3 drops of the dilution were also introduced to the ATB medium for the testing of antimicrobial susceptibility. In addition, a streak isolation from non-diluted urine was performed on Columbia blood and Drigalski agars: this was a control inoculation.

\section{Results}

For the 200 urine specimens studied with both methods, 176 identifications and 174 antimicrobial susceptibility tests were performed with the rapid method (two strains did not grow on ATB medium with either the conventional or the rapid method). In 24 cases the results of the rapid method were discarded because there were mixtures of different Gram negative bacilli. Table 1 shows the identification of the different strains. There was complete agreement between the two methods. As the ATB Gram negative strip contains 14 antibiotics, comparison was carried out on 2436 determinations. Two major discrepancies only were observed in the cases of Proteus mirabilis and Escherichia coli, the two strains being resistant to cefazolin with the conventional method and sensitive with the rapid method. There were a few minor discrepancies in 155 of 2436 determinations: in 48 cases
Table 1 Distribution among species of bacteria used for comparison of both methods

\begin{tabular}{lc}
\hline Species & No of strains \\
\hline & \\
Escherichia coli & 114 \\
Citrobacter freundii & 3 \\
Klebsiella pneumoniae & 23 \\
Enterobacter cloacae & 7 \\
Serratia marcescens & 2 \\
Proteus mirabilis & 19 \\
Proteus vulgaris & 2 \\
Providencia stuartii & 3 \\
Morganella morganii & 1 \\
Pseudomonas aeruginosa & 1 \\
Acinetobacter calcoaceticus & 1 \\
Total & 176 \\
\hline
\end{tabular}

the rapid method yielded "more resistant" results than the conventional method (intermediate response with rapid method and sensitive response with conventional method in 32 cases, resistant with rapid method, intermediate with conventional method in 16), and in 107 cases the rapid method gave a higher sensitivity (sensitive response with rapid method and intermediate response with conventional method in 59 cases; intermediate with rapid method and resistant with conventional method in 48). Analysis of discrepancies for each antibiotic (Table 2) indicates that the distribution was fairly uniform and not specific for a single antibiotic. The number of discrepancies did not seem to vary with the genus, but the small number of strains studied did not allow valuable comparisons to be made.

Table 2 Correlation of antimicrobial susceptibility tests between rapid and conventional methods (figures in parentheses indicate number)

\begin{tabular}{|c|c|c|c|c|c|}
\hline \multirow[t]{3}{*}{ Antibiotic } & \multicolumn{4}{|c|}{ Percentage of minor discrepancies* } & \multirow{3}{*}{$\begin{array}{l}\text { Percentage of } \\
\text { agreement }\end{array}$} \\
\hline & \multicolumn{4}{|l|}{ Distribution } & \\
\hline & $R / I \dagger$ & $I / S$ & $I / R$ & $S / I$ & \\
\hline $\begin{array}{l}\text { Ampicillin } \\
\text { Ticarcillin } \\
\text { Cefazolin } \\
\text { Cefotaxim } \\
\text { Cefsulodin } \\
\text { Gentamicin } \\
\text { Dibekacin } \\
\text { Amikacin } \\
\text { Doxycyclin } \\
\text { Chloramphenicol } \\
\text { Colistin } \\
\text { Nalidixic acid } \\
\text { Pipemidic acid } \\
\text { Co-trimoxazole } \\
\text { Total }\end{array}$ & $\begin{array}{l}0.0(0) \\
0.0(0) \\
1.14(2) \\
0.0(0) \\
2.29(4) \\
0.0(0) \\
0.57(1) \\
0.0(0) \\
2.29(4) \\
0.0(0) \\
0.57(1) \\
0.57(1) \\
1.14(2) \\
0.57(1) \\
\quad(16)\end{array}$ & $\begin{array}{l}2.30(4) \\
1 \cdot 14(2) \\
1 \cdot 14(2) \\
0.57(1) \\
1 \cdot 14(2) \\
1.72(3) \\
1.72(3) \\
1 \cdot 72(3) \\
0.57(1) \\
1 \cdot 14(2) \\
1.72(3) \\
0.57(1) \\
1.72(3) \\
1.14(2) \\
(32)\end{array}$ & $\begin{array}{l}2.87(5) \\
1.72(3) \\
4.60(8) \\
0.57(1) \\
7.47(13) \\
0.57(1) \\
2.29(4) \\
0.0(0) \\
4.02(7) \\
0.57(1) \\
0.0(0) \\
0.57(1) \\
1.72(3) \\
0.57(1) \\
\quad(48)\end{array}$ & $\begin{array}{l}4 \cdot 02(7) \\
1 \cdot 14(2) \\
9 \cdot 19(16) \\
1 \cdot 14(2) \\
1 \cdot 14(2) \\
1 \cdot 14(2) \\
2 \cdot 87(5) \\
0 \cdot 57(1) \\
1 \cdot 14(2) \\
6 \cdot 32(11) \\
0 \cdot 0(0) \\
1 \cdot 72(3) \\
2 \cdot 29(4) \\
1 \cdot 14(2) \\
\quad(59)\end{array}$ & $\begin{array}{l}90 \cdot 8 \\
96 \\
83.93 \\
97 \cdot 12 \\
87.96 \\
96.57 \\
92.55 \\
97.71 \\
91.98 \\
91.97 \\
97.71 \\
96.57 \\
93.13 \\
96.58\end{array}$ \\
\hline
\end{tabular}

*Only two major discrepancies in the study.

+R/I Response: Resistant with rapid method. Intermediate with conventional method.

I/S Response: Intermediate with rapid method. Sensitive with conventional method.

I/R Response: Intermediate with rapid method. Resistant with conventional method.

S/I Response: Sensitive with rapid method. Intermediate with conventional method. 


\section{Discussion}

The results of this study show that the Gram negative bacilli most often responsible for urinary infection can be accurately identified directly from urine in 24 hours, even at the species level, using the API $20 \mathrm{E}$ system, with a $100 \%$ correlation with the conventional technique.

Other observers have described a positive correlation, depending on the method used: $94 \%^{1}$ with the FPS Pathotec, $94 \%{ }^{2}$ and $95 \%{ }^{3}$ with the Micro ID system, and $92 \%{ }^{6}$ with the Automicrobic system. Our correlation of $100 \%$ can be explained by the $1 / 500$ urine dilution that suppresses contaminating bacteria: even in small numbers, these are able to change one or several reactions in the API $20 \mathrm{E}$ system. Indeed, isolates obtained from streaks of non-diluted urine allowed us to grow small numbers $\left(<10^{4} / \mathrm{ml}\right)$ of Streptococcus five times and Proteus mirabilis three times, but their influence was eliminated by $1 / 500$ dilution. The dilution was determined by the results of a preliminary study on the detection level of API 20 E: by dilution and counting on solid media we estimated up to $10^{6}$ to $10^{7}$ bacteria per $\mathrm{ml} /$ inoculum, the figure suggested by the manufacturer under normal conditions. Thus in another way we established that API $20 \mathrm{E}$ inoculum can be reduced to $10^{2}$ bacteria per $\mathrm{ml}$, while still yielding good results. For this reason the rapid technique used here seems applicable to all cases of urinary tract infection, because an infected urine contains between $10^{5}$ and $10^{8}$ viable bacteria per $\mathrm{ml}$.

The method proposed here, however, failed in two cases, as it did in other studies. ${ }^{1-511}$ Urine specimen containing between $10^{4}$ and $10^{5}$ bacteria $/ \mathrm{ml}$ were not always detected, and in these cases the results of the classical method were more reliable. This situation occurred once during our study with a urine specimen that contained $10^{4}$ Pmirabilis per $\mathrm{ml}$ as a result of previous treatment with antibiotics.

When urine contained a mixture of Gram negative bacteria, which cannot be discerned on direct examination, the identification strip was then illegible and the antimicrobial susceptibility test atypical. This situation occurred 24 times during our study, and the only valuable results were obtained with the conventional method.

The urines were screened by Gram staining performed without centrifugation, according to Lewis and Alexander ${ }^{12}$ and Heinze et al. ${ }^{1}$ Two hundred of 1152 urines examined were selected for the rapid method, while 228 urines yielded Gram negative organisms by the conventional method, making a correlation of $85 \%$. These results agree with those of other authors: Thrupp et al $^{6}$ obtained a correlation of $89 \%$. It is clear that Gram staining is not the most sensitive method for detecting organisms in infected urines; it is, however, the most simple.

A correlation of $94 \%$ was obtained for the antimicrobial susceptibility test, comparable with or better than that of other studies. ${ }^{1-3511}$ There were only two major discrepancies out of 2436 comparisons $(0.08 \%)$ in our study, whereas discrepancies of 3 to $4 \%$ have been observed by other authors. ${ }^{1-3}$ Table 2 shows that the minor discrepancies occurred in either direction: one method does not systematically yield "more sensitive" results or "more resistant" results than the other. In a previous study the repeatability of the ATB API method was $97 \%{ }^{10}$ and the correlation of $94 \%$ observed here is very close to this value. Moreover, this study was performed on clinical strains, some of which had been obtained from patients in hospital for a long period who had multiple antibiotic resistance. In such cases more discrepancies occur than with the more sensitive strains usually responsible for infection in the lower urinary tract.

These particular patients accounted for our choice of antibiotics, as they are not all specific for urinary infection. The antimicrobial susceptibility testing could be performed similarly, using the ATB urinary system (ATB-UR), the products of which are better adapted to less severe infections. Patients who have been in hospital for long periods may also present more polymicrobial infections with Gram negative bacteria, as was the case in 24 of 200 .

These observations support the high reliability of the method proposed here. The protocol we suggest is as follows: cytological examination of the urine specimen and Gram staining; inoculation with a $1 / 100$ dilution according to the conventional method described in this paper; if the urine contains Gram negative bacilli on direct examination, without cocci, inoculation of both the API $20 \mathrm{E}$ system and ATBGram medium with 1/500 dilution of urine according to the rapid method should be used; examination of results 24 hours later, after comparison of the responses from API 20 E and ATB Gram negative systems with the appearance of isolates to ascertain the purity of the strain and the quality of the results. In case of uncertainty with the rapid method the simultaneously started conventional method will still yield results at 48 hours.

\section{Conclusion}

Our method is slightly longer ( 24 hours) than those using Autobac or Automicrobic systems (four to 13 hours), it is, however, more reliable $(100 \%$ correlation for identification $v 90-95 \%$ and $0.08 \%$ major discrepancies $v 3-4 \%$ according to other authors). We put forward this method as an intermediate method between the earlier rapid methods and the classical 
methods, because it seems to be as reliable as the classical methods. We must, however, point out that, as with all the rapid methods, it can be applied only to monomicrobial infections and must be used with precision. It does not need a lot of capital investment, and due to the accuracy of its results, it should find widespread acceptance as a standard laboratory method.

We thank the members of the laboratory for excellent technical help.

\section{References}

${ }^{1}$ Heinze PA, Thrupp LD, Anselmo CR. A rapid (4-6 hours) urineculture system for direct identification and direct antimicrobial susceptibility testing. Am J Clin Pathol 1979;71:177-83.

${ }^{2}$ Jenkins RD, Hale DC, Matsen JM. Rapid semi-automated screening and processing of urine specimens. J Clin Microbiol 1980;11:220-5.

${ }^{3}$ Kelly MT, Hale DC, Matsen JM. Rapid identification by the micro-ID system of enterobacteriaceae detected by urine screening. J Clin Microbiol 1981;14:295-7.

${ }^{4}$ Nicholson DP, Hoepke JA. The automicrobic system for urines. $J$
Clin Microbiol 1979;10:823-33.

${ }^{5}$ Tilton RE, Tilton RC. Automated direct antimicrobial susceptibility testing of microscopically screening urine cultures. $J$ Clin Microbiol 1980;11:157-61.

${ }^{6}$ Thrupp LD, Heinze PD, Pezzlo MA. Direct, rapid urine cultures: same day reporting of quantitation, speciation and antimicrobial susceptibility tests. In: Balows $\mathrm{A}$, ed. ASM symposium of automation in clinical microbiology. New York: Pfizer Diagnostics, 1976:17-22.

${ }^{7}$ Aldridge KE, Garner BB, Clark SJ, Matsen JM. Comparison of micro ID, API $20 \mathrm{E}$ and conventional media systems in identification of enterobacteriaceae. $J$ Clin Microbiol 1978;7:507-13.

${ }^{8}$ Holmes B, Willcox WR, Lapage SP. Identification of enterobacteriaceae by the API 20 E System. J Clin Pathol 1978;31:22-30.

${ }^{9}$ Smith PB, Tomfohrde KM, Rhoden DL, Balows A. API System: a multitube micromethod for identification of Enterobacteriaceae. Appl Microbiol 1972;24:449-52.

${ }^{10}$ Dupeyron C, Vuillemin N, Bories C, Leluan C. Comparison de trois méthodes d'antibiogramme. Ann Biol Clin 1981;39:61-8.

${ }^{11}$ Wadke M, McDonnell C, Ashton JK. Rapid processing of urine specimens by urine screening and the automicrobic system. $J$ Clin Microbiol 1982;16:668-72.

${ }^{12}$ Lewis JF, Alexander J. Microscopy of stained urine smears to determine the need for quantitative culture. J Clin Microbiol 1976;4:372-4.

Requests for reprints to: Dr Catherine Dupeyron, Laboratoire de Bacteriologie, Hôpital Albert Chenevier, Rue de Mesly 40, 94010 Creteil, France. 